\title{
Sports and Recreational Activities following Total Ankle Replacement
}

\author{
Federico Giuseppe Usuelli ${ }^{1}$ Andrea Pantalone ${ }^{2}$ Camilla Maccario ${ }^{1}$ Matteo Guelfi ${ }^{2}$ Vincenzo Salini ${ }^{2}$ \\ ${ }^{1}$ Casco, Foot and Ankle Unit, IRCCS Galeazzi, Milan, Italy \\ ${ }^{2}$ Orthopaedic and Traumatology Division, Department of Medicine \\ and Science of Aging, University G. d'Annunzio, Chieti-Pescara, \\ Chieti, Italy

\begin{abstract}
Address for correspondence Matteo Guelfi, MD, Orthopaedic and Traumatology Division, Department of Medicine and Science of Aging, University G. d'Annunzio, Chieti-Pescara, Via dei Vestini 35,
\end{abstract} \\ 66013 Chieti, Italy (e-mail: matteogue@hotmail.com).
}

Joints 2017;5:12-16

\begin{abstract}
Purpose In literature, there is a controversy regarding whether patients who have undergone total ankle replacement (TAR) can participate in sports and recreational activities. The purpose of this study was to report change in sports activity level after TAR.

Methods A retrospective study was performed, enrolling 76 patients with symptomatic end-stage ankle arthritis who underwent TAR from May 2011 to October 2014. Patients were mainly males $(44 / 76 ; 58 \%)$ and 56 years old on average (range: $22.3-$ 79.6 years) at the time of surgery. They were treated with mobile-bearing prosthesis implanted with an anterior approach. Patients were evaluated preoperatively and 12 months postoperatively. Pain and function were assessed using the American Orthopaedic Foot and Ankle Society (AOFAS) ankle and hindfoot score, the visual analog scale (VAS) pain score, and the 12-Item Short Form Health Survey (SF-12) - physical component summary (PCS) and mental component summary (MCS). Activity level was assessed with the University of California at Los Angeles (UCLA) activity scale.

Results At 12 months postoperatively, statistically significant increase was reported for AOFAS scores (from $32.8 \pm 12.7$ preoperatively to $72.6 \pm 13.3 ; p<0.001$ ), SF-12 PCS (from $34.3 \pm 5.1$ preoperatively to $45.4 \pm 6.4 ; p<0.001$ ), and SF-12 MCS (from $39.8 \pm 7.5$ preoperatively to $51.4 \pm 6.1 ; p<0.001$ ). A statistically significant decrease
Keywords
- ankle
- arthroplasty
- osteoarthritis
- replacement
- sport was detected in VAS pain score (from $8.7 \pm 1.6$ preoperatively to $2.2 \pm 1.6 ; p<0.001$ ). The UCLA activity levels increased significantly from $2.4 \pm 0.8$ to $6.3 \pm 2.3(p<0.001)$. Conclusion Pain and function significantly improved in patients affected by ankle osteoarthritis, who underwent TAR, at 1-year follow-up. In addition, activity level showed a significant increase respect to preoperative condition.

Level of Evidence Level IV, retrospective case series.
\end{abstract}

\section{Introduction}

Ankle osteoarthritis (OA) incidence is significantly lower than knee and hip arthritis. ${ }^{1}$ It is mostly posttraumatic (65-80\% of the cases), and this may explain a different epidemiology with a younger population affected. ${ }^{2}$ Historically, design issues are related to early failures of total ankle replacement (TAR) rather than patient age or high-impact activities. Furthermore, it has been showed that TAR is a high demanding surgery with a long learning curve. This may contribute to raise the rate of failures in small cohorts, rather than patient age and high-impact activities. In the past, ankle arthrodesis was considered the first surgical treatment for end-stage arthritis of the ankle. However, during the years,
DOI http://dx.doi.org/ $10.1055 / \mathrm{s}-0037-1601408$ ISSN 2282-4324.
Copyright @ 2017 Georg Thieme Verlag KG Stuttgart · New York
License terms

()(1) $\odot \circledast$ 
some important disadvantages related to this surgery became clear: compensatory overload, gait change, high rates of nonunion, long rehabilitation period, and eventual development of adjacent joint OA. ${ }^{3}$ These led to a new increasing interest in TAR, inducing important development in implants design and materials.

Mobile-bearing implants represented a revolutionary and successful step forward in Europe. With their consistent use, it has been clarified that primary implant stability should be the main focus of the surgeon rather than any cement fixation. Kofoed and Lundberg-Jensen ${ }^{4}$ evaluated early generation cemented and uncemented mobile-bearing TAR and concluded that the results of TAR were equivalent in patients younger and older than 50 years, pointing out cement fixation as a major reason for failures rather than patient age or high-impact activities. Recently, some authors proposed TAR as the gold standard treatment for ankle OA, even in young and active patients. Demetracopoulos et $\mathrm{al}^{5}$ claimed comparable results with TAR in younger ( $<55$ years) and older patients ( $>70$ years) at early follow-up. The incidences of complications and the revision rate were similar between the two groups. However, the authors did not describe physical activities allowed for these patients. There is still a controversy regarding whether patients who underwent TAR can participate in sport and recreational activities. ${ }^{6}$

Today, there is a new trend toward better results in terms of quality of life, and physical and athletic ability after TAR than after tibiotalar arthrodesis. ${ }^{7}$

The aim of this study was to analyze sports activity before and after TAR. The hypothesis of the study was that sports activity of patients affected by ankle OA increases after TAR.

\section{Methods}

This study is a retrospective review of 76 patients who were treated with Hintegra total ankle prosthesis (Newdeal, Lyon, France; Integra LifeSciences, Plainsboro, New Jersey, United States) between May 2011 and October 2014. All patients were followed up 12 months after surgery. The indications for symptomatic end-stage ankle OA were posttraumatic OA (70 patients), revision surgery of an osteochondral lesion procedure failure (one patient), posterior tibial tendon dysfunction (PTTD) stage IV according to the Bluman-Myerson ${ }^{8}$ classification (one patient), drop foot (one patient), and rheumatoid arthritis (three patients).

All patients, except for those with the rheumatoid arthritis and drop foot, had prior ankle surgeries: patients with posttraumatic OA previously had fixation of fractures and, in a few cases, removal of internal fixation, the patient with osteochondral lesion underwent two arthroscopic treatments performed by other surgeons, and the patient with PTTD was previously treated with subtalar and talonavicular arthrodesis.

All TAR procedures were performed by one of the coauthors according to the technique by Barg et $\mathrm{al}^{9}$ : an anterior ankle approach of 10 to $12 \mathrm{~cm}$ length was made. The retinaculum was dissected along the lateral border of the

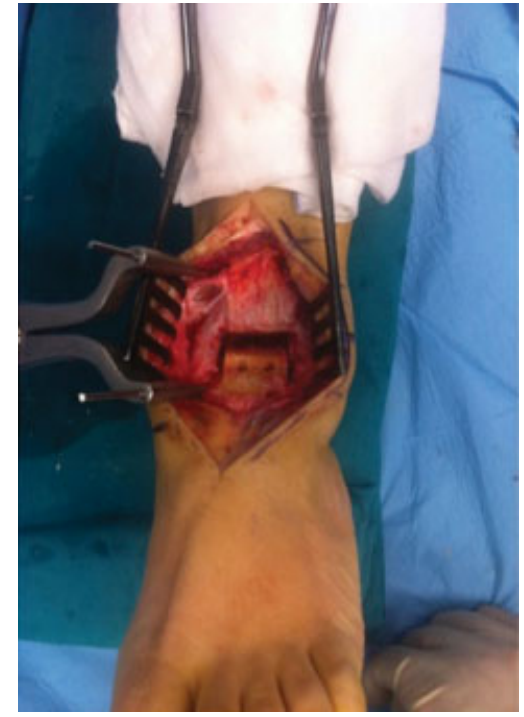

Fig. 1 Anterior ankle approach for total ankle replacement.

anterior tibial tendon, avoiding the neurovascular bundle that runs behind the long extensor hallucis tendon ( - Fig. 1).

Patients were evaluated preoperatively and 12 months after surgery. Pain and function were assessed using the American Orthopaedic Foot and Ankle Society (AOFAS) ankle and hindfoot score, the visual analog scale (VAS) pain score, and the 12-Item Short Form Health Survey (SF-12) - physical component summary (PCS) and mental component summary (MCS). Furthermore, the patients were radiologically examined preoperatively and at follow-up using standard weight-bearing radiographs (anteroposterior and lateral views $)^{10}$ and the Saltzman alignment view ${ }^{11}$ (-Fig. 2).

Pre- and postoperative activity level was determined using the University of California at Los Angeles (UCLA) activity scale. ${ }^{12}$ The UCLA scale is based on the patient's ranking of her/his level of physical activity. The score ranges from 1 to 10 . The patient had to indicate her/his most appropriate activity level, with level 1 defined as "no physical activity, dependent on others" and level 10 defined as "regular participation in impact sports."13,14

All data were expressed as mean and standard deviation. Statistical analysis was performed with MATLAB statistical toolbox version 2008 (MathWorks, Natick, Massachusetts, United States). Paired Student's $t$-test was used to compare baseline and follow-up values. $P$-values less than 0.05 were considered statistically significant.

\section{Results}

A total of 76 patients were included in this review. Patients were mainly males $(44 / 76 ; 58 \%)$, were 56 years old on average (range: 22.3-79.6 years) at the time of surgery, and were treated with Hintegra prosthesis (Allegra Orthopaedics, Lane Cove West, NSW, Australia) with an anterior approach.

Forty right and 36 left TARs were performed. The prosthesis components sizes were mismatched (talus component 

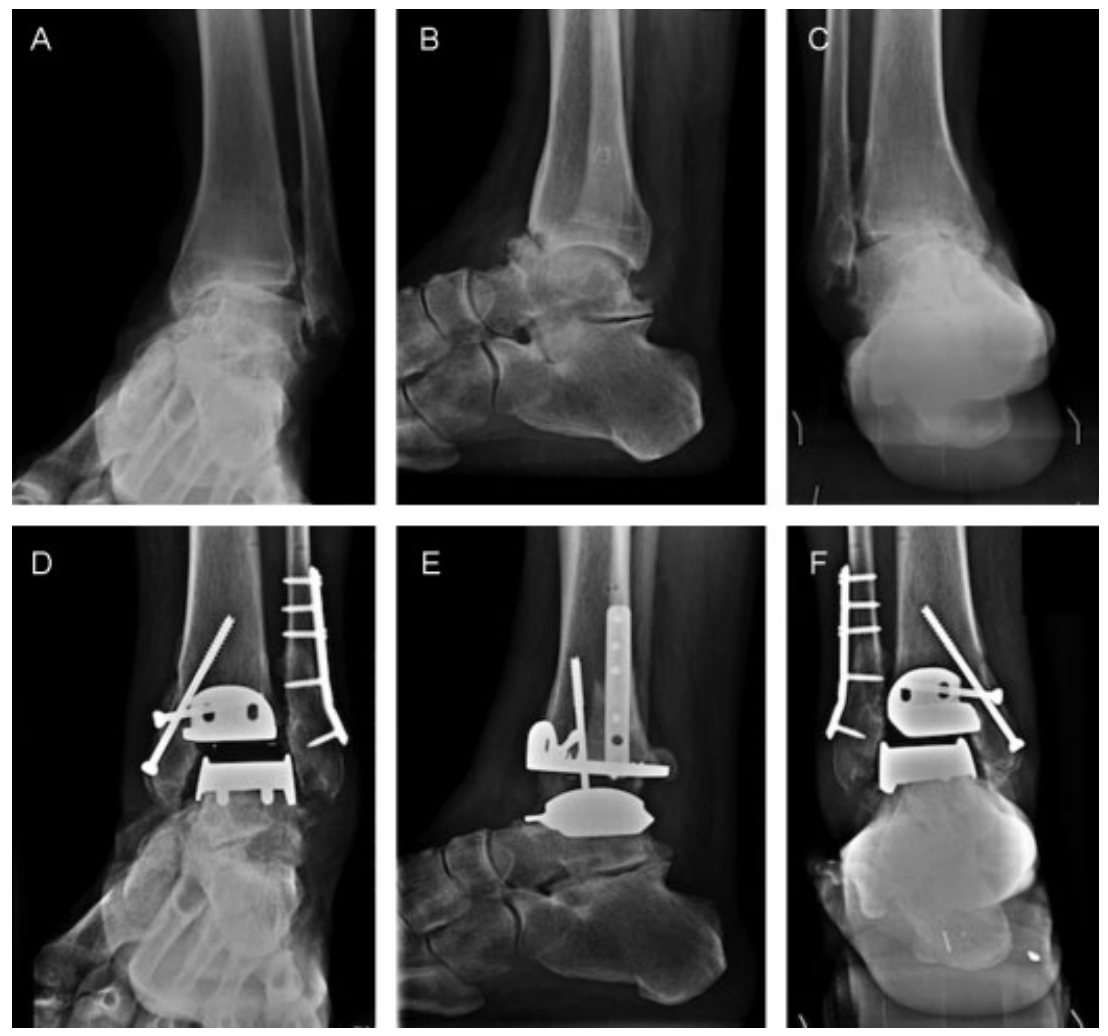

Fig. 2 Painful end-stage varus osteoarthritis of the left ankle in a 54-year-old man. Preoperative standard weight-bearing X-rays in (A) anteroposterior and (B) lateral views. (C) Saltzman alignment view shows a significant varus malalignment of the heel. Postoperative radiographs in (D) anteroposterior, (E) lateral, and (F) Saltzman alignment view show a significant correction of the malalignment of the heel.

size smaller than tibial component size) in 32 patients. Nineteen patients underwent the following concurrent operative procedure at the time of TAR: Achilles tendon lengthening (seven patients), realignment calcaneal osteotomy (three patients), and subtalar arthrodesis (three patients). Ligament balancing was performed as required through an isolated deltoid release (one patient), a medial malleolar lengthening osteotomy (three patients), and a combination of medial malleolar lengthening osteotomy, fibular shortening osteotomy, and peroneus longus to brevis transfer (two patients).

At 12 months postoperatively, there was a statistically significant increase in AOFAS scores from $32.8 \pm 12.7$ preoperatively to $72.6 \pm 13.3(p<0.001)$, in SF-12 PCS from 34.3 \pm 5.1 preoperatively to $45.4 \pm 6.4(p<0.001)$, and in the SF12 MCS from $39.8 \pm 7.5$ preoperatively to $51.4 \pm 6.1$ $(p<0.001)$. In addition, there was also a statistically significant decrease in the VAS pain score from $8.7 \pm 1.6$ preoperatively to $2.2 \pm 1.6(p<0.001)$. The UCLA activity levels increased significantly from $2.4 \pm 0.8$ to $6.3 \pm 2.3$ $(p<0.001)(-$ Table 1$)$.

Before surgery, $11.7 \%$ of the patients were active in sports; after surgery, this percentage rose to $49.4 \%$. The most frequent sports activities were jogging, dancing, biking, and skiing; a fraction of patients $(n=14)$ participated in impact sports such as jogging (13 patients) and martial arts (1 patient) in spite of adverse medical advice (-Table 2).
Table 1 UCLA score

\begin{tabular}{|l|l|l|l|l|}
\hline & \multicolumn{2}{|l|}{ Pre-Operative } & \multicolumn{2}{l|}{} \\
\hline & \multicolumn{2}{l|}{$\begin{array}{l}\text { Arthritic } \\
\text { Condition }\end{array}$} & \multicolumn{2}{l|}{ Post- TAR } \\
\hline UCLA Score & $\%$ & $\mathbf{N}^{\circ}$ of Pts & $\%$ & $\mathbf{N}^{\circ}$ of Pts \\
\hline 1 & 5,3 & 4 & 0 & 0 \\
\hline 2 & 61,8 & 47 & 7,9 & 6 \\
\hline 3 & 23,7 & 18 & 7,9 & 6 \\
\hline 4 & 6,6 & 5 & 6,6 & 5 \\
\hline 5 & 2,6 & 2 & 9,2 & 7 \\
\hline 6 & 0 & 0 & 17,1 & 13 \\
\hline 7 & 0 & 0 & 14,5 & 11 \\
\hline 8 & 0 & 0 & 18,4 & 14 \\
\hline 9 & 0 & 0 & 14,5 & 11 \\
\hline 10 & 0 & 0 & 3,9 & 3 \\
\hline Total & 100 & 76 & 100 & 76 \\
\hline Mean UCLA Score & 2,4 & & 6,3 \\
\hline
\end{tabular}

\section{Discussion}

Ankle OA has a deeply different epidemiology from hip and knee. These may be related to biomechanics reasons ${ }^{15}$ : the loading area of the ankle is approximately one-third smaller 
Table 2 Recommendations of sports activities

\begin{tabular}{|l|l|l|}
\hline Recommended & Possible & $\begin{array}{l}\text { Not } \\
\text { recommended }\end{array}$ \\
\hline Aerobic activity & $\begin{array}{l}\text { Cross-country } \\
\text { skiing }\end{array}$ & Basketball \\
\hline Cycling & Sailing & Beach volleyball \\
\hline Dancing & Alpine skiing & Climbing \\
\hline Golf & Tennis & Horseback riding \\
\hline $\begin{array}{l}\text { Hiking or nordic } \\
\text { walking }\end{array}$ & Hunting & Ice hockey \\
\hline Swimming & & $\begin{array}{l}\text { Ice skating and } \\
\text { skating }\end{array}$ \\
\hline & & Jogging \\
\hline & & Martial arts \\
\hline & & Mountain biking \\
\hline & & $\begin{array}{l}\text { Paragliding and } \\
\text { skydiving }\end{array}$ \\
\hline & & Rugby \\
\hline & & Soccer \\
\hline & & Volleyball \\
\hline & & Water ski \\
\hline & & Windsurfing \\
\hline
\end{tabular}

than the corresponding ones for knee or hip ${ }^{16}$; the thinner (1-2 mm) cartilage layer of the ankle offer a far more resilient tissue than those of the other joints. ${ }^{17}$ Posttraumatic changes are the main reasons for developing ankle OA ( 65-80\%).

Ankle trauma is more likely to affect younger population. This can also explain a younger population for ankle OA, which usually affect higher demanding patients if compared with hip and knee. In this respect, as a consequence of the impressive progress achieved in ankle prosthesis designs and clinical outcome following TAR, ankle arthrodesis is no longer the standard treatment for end-stage ankle OA. ${ }^{18}$

In our study, patient's recovery and joint restoration in terms of balance and movement suggest that TAR may be considered the golden standard at least for highly active patients. This statement finds a confirmation in our UCLA scale results. The UCLA activity score is described as a valid instrument for assessment of activity levels in patients undergoing total joint arthroplasties ${ }^{13}$ : we detected a significant increase in UCLA activity levels from 2.4 to 6.3.

The most frequent sports activities were jogging, dancing, biking, and skiing; a group of patients ${ }^{14}$ participated in impact sports such as jogging ${ }^{13}$ and martial arts ${ }^{1}$ in spite of adverse medical advice.

Despite of our early results, high-impact sports activities may increase polyethylene wear ${ }^{19}$ and the incidence of the fracture of polyethylene ${ }^{20}$ at a long-term follow-up. In addition, young age, high body mass index, and poor bone quality are additional risk factors that led orthopaedic surgeons to remain more cautious and restrictive. ${ }^{6}$

Nevertheless, the first clinical study on sport and recreation activities in patients who underwent TAR published in
2006 found that the rate of sports participation increased from $36 \%$ preoperatively to $56 \%$ postoperatively. ${ }^{21}$ Furthermore, some authors pointed out that patients active in sports were not at higher risk for failure compared with other groups $^{18}$; Naal et al reported that neither sports participation nor activity levels were identified as risk factors for development of periprosthetic radiolucencies. ${ }^{14}$

A critical factor in sport activities is the ankle range motion. Although the major advantages of TAR versus ankle arthrodesis is the preservation of ankle mobility, there is still no consensus whether patients with TAR have better results in sport activities than patients with ankle fusion. In fact, Schuh et $\mathrm{al}^{22}$ revealed no significant difference between these two groups concerning activity levels, participation in sports activities, and UCLA and AOFAS scores. Anyway, the patients after ankle arthrodesis significantly decreased their activities. It may be related to the loss of movement due to the degenerative changes in the ipsilateral, tarsometatarsal, and subtalar joints. ${ }^{23-25}$

Recently, Flavin et $\mathrm{al}^{26}$ demonstrated that patients with TAR showed higher speed during walk. In fact, the restored functionality of the ankle allowed a comparatively increased stride length and faster cadence, as well as more normalized first and second rockers at the gait analysis.

Bonnin et $\mathrm{al}^{27}$ in their retrospective series of 140 mobilebearing ankle arthroplasties showed that TAR improved the quality of life and that return to recreational activities was generally possible but the return to impact sport was rarely possible. As our results suggest, less pain and better range of motion can encourage patients to increase their participation in sports (including high impact ones) and recreation activities, but this observation should be checked at a longer follow-up.

Although TAR has become an increasingly popular procedure to treat symptomatic and recalcitrant ankle arthritis, orthopaedic surgeons have little guidance concerning physical activity and sport participation recommendations following this procedure.

Our results suggest, though preliminary, that TAR is possibly a better choice with respect to arthrodesis, at least for highly active individuals. This consideration comes from both patients' perception and, more objectively, the reduced stress imposed by TAR to the nearest joints with respect to OA.

To date, further studies on the durability and complications of prostheses, as well as patient activity and return to specific sports and activities after surgery, are needed to reach a unique guideline based on stronger levels of evidence.

\section{References}

1 Huch K. Knee and ankle: human joints with different susceptibility to osteoarthritis reveal different cartilage cellularity and matrix synthesis in vitro. Arch Orthop Trauma Surg 2001;121(06):301-306

2 Horisberger M, Valderrabano V, Hintermann B. Posttraumatic ankle osteoarthritis after ankle-related fractures. J Orthop Trauma 2009;23(01):60-67

3 Boobbyer GN. The long-term results of ankle arthrodesis. Acta Orthop Scand 1981;52(01):107-110 
4 Kofoed H, Lundberg-Jensen A. Ankle arthroplasty in patients younger and older than 50 years: a prospective series with long-term follow-up. Foot Ankle Int 1999;20(08):501-506

5 Demetracopoulos CA, Adams SB Jr, Queen RM, DeOrio JK, Nunley JA II, Easley ME. Effect of age on outcomes in total ankle arthroplasty. Foot Ankle Int 2015;36(08):871-880

6 Macaulay AA, VanValkenburg SM, DiGiovanni CW. Sport and activity restrictions following total ankle replacement: a survey of orthopaedic foot and ankle specialists. Foot Ankle Surg 2015; 21(04):260-265

7 Dalat F, Trouillet F, Fessy MH, Bourdin M, Besse JL. Comparison of quality of life following total ankle arthroplasty and ankle arthrodesis: retrospective study of 54 cases. Orthop Traumatol Surg Res 2014;100(07):761-766

8 Bluman EM, Title CI, Myerson MS. Posterior tibial tendon rupture: a refined classification system. Foot Ankle Clin 2007;12(02): 233-249, v

9 Barg A, Knupp M, Henninger HB, Zwicky L, Hintermann B. Total ankle replacement using HINTEGRA, an unconstrained, threecomponent system: surgical technique and pitfalls. Foot Ankle Clin 2012;17(04):607-635

10 Hintermann B, Valderrabano V, Dereymaeker G, Dick W. The HINTEGRA ankle: rationale and short-term results of 122 consecutive ankles. Clin Orthop Relat Res 2004;(424):57-68

11 Saltzman CL, el-Khoury GY. The hindfoot alignment view. Foot Ankle Int 1995;16(09):572-576

12 Zahiri CA, Schmalzried TP, Szuszczewicz ES, Amstutz HC. Assessing activity in joint replacement patients. J Arthroplasty 1998; 13(08):890-895

13 Naal FD, Impellizzeri FM, Leunig M. Which is the best activity rating scale for patients undergoing total joint arthroplasty? Clin Orthop Relat Res 2009;467(04):958-965

14 Naal FD, Impellizzeri FM, Loibl M, Huber M, Rippstein PF. Habitual physical activity and sports participation after total ankle arthroplasty. Am J Sports Med 2009;37(01):95-102

15 Labib SA, Raikin SM, Lau JT, et al. Joint preservation procedures for ankle arthritis. Foot Ankle Int 2013;34(07):1040-1047
16 Kimizuka M, Kurosawa H, Fukubayashi T. Load-bearing pattern of the ankle joint. Contact area and pressure distribution. Arch Orthop Trauma Surg 1980;96(01):45-49

17 Thomas RH, Daniels TR. Ankle arthritis. J Bone Joint Surg Am 2003;85-A(05):923-936

18 Hörterer H, Miltner O, Müller-Rath R, Phisitkul P, Barg A. Sports activity in patients with total ankle replacement. Sport-OrthopTraumatol 2015;31:34-40

19 Harris NJ, Brooke BT, Sturdee S. A wear debris cyst following S.T.A R. Total Ankle Replacement-surgical management. Foot Ankle Surg 2009;15(01):43-45

20 Scott AT, Nunley JA. Polyethylene fracture following STAR ankle arthroplasty: a report of three cases. Foot Ankle Int 2009;30(04): 375-379

21 Valderrabano V, Pagenstert G, Horisberger M, Knupp M, Hintermann B. Sports and recreation activity of ankle arthritis patients before and after total ankle replacement. Am J Sports Med 2006; 34(06):993-999

22 Schuh R, Hofstaetter J, Krismer M, Bevoni R, Windhager R, Trnka HJ. Total ankle arthroplasty versus ankle arthrodesis. Comparison of sports, recreational activities and functional outcome. Int Orthop 2012;36(06):1207-1214

23 Glazebrook M. End-stage ankle arthritis: magnitude of the problem and solutions. Instr Course Lect 2010;59: 359-365

24 Fuchs S, Sandmann C, Skwara A, Chylarecki C. Quality of life 20 years after arthrodesis of the ankle. A study of adjacent joints. J Bone Joint Surg Br 2003;85(07):994-998

25 Buchner M, Sabo D. Ankle fusion attributable to posttraumatic arthrosis: a long-term followup of 48 patients. Clin Orthop Relat Res 2003;(406):155-164

26 Flavin R, Coleman SC, Tenenbaum S, Brodsky JW. Comparison of gait after total ankle arthroplasty and ankle arthrodesis. Foot Ankle Int 2013;34(10):1340-1348

27 Bonnin MP, Laurent JR, Casillas M. Ankle function and sports activity after total ankle arthroplasty. Foot Ankle Int 2009;30(10): 933-944 